direkte Demokratie in der Masse der Abstimmungen „als ungeeignet als Instrument gegen die Dominanz der Parteien“ (S. 323). Gegen Jung und ihre Systemtyp-Erklärung pointiert Hornig, „dass die wahre Logik direkter Demokratie jene der repräsentativen Sphäre (mit nur wenigen Ausflüchten) ist“ (S. 335).

Leider schmälert der Verfasser dieser bei Ralf Kleinfeld in Osnabrück entstandenen Dissertation seine Verdienste, wenn er formuliert, „das Bild, das von direkter Demokratie allenthalben verbreitet wird, (müsse) korrigiert werden. Volksabstimmungen sind nur sehr selten ein Korrektiv der repräsentativen Akteure“ (S. 330). Oder gar, seine Ergebnisse lieferten „einen bislang fehlenden Realismus für den Umgang mit direkter Demokratie“ (S. 335).

Tilman Evers hat schon vor Jahrzehnten in einem eher geisteswissenschaftlichen Zugriff bezweifelt, dass direkte Demokratie „ein wirksames Mittel gegen den Parteien-Absolutismus wäre“ 1 . Volksentscheide könnten sich „nur punktuell und begrenzt unterscheiden von dem, was heute im parlamentarischen Verfahren an Resultaten durchsetzbar ist. Dieser geringe Unterschied kann im Einzelfall wichtig sein “2. In seinem letzten Satz schwang allerdings ein bisschen Hoffnung mit, während Hornig mit der Rede von „Störfaktor“ und „Denkzettel-Abstimmungen“ schon sprachlich die Parteienperspektive verinnerlicht hat.

Otmar Jung

1 Tilman Evers, Mehr Demokratie durch Volksentscheid?, in: Kritische Justiz, 19. Jg. (1986), H. 4, S. $423-433$, S. 429.

2 Ders., Zivilgesellschaft und direkte Demokratie in gesamtdeutscher Verfassung, in: Staat und Recht, 39. Jg. (1990), H. 4, S. 931 - 945, S. 942.

\title{
Zweite Kammern: gehaltvolle Analysen, editorische und redaktionelle Mängel
}

\section{Leunig, Sven (Hrsg.): Handbuch Föderale Zweite Kammern, Verlag Barbara Budrich, Opladen} I Farmington Hills 2009, 313 Seiten, € 48,-.

Riescher, Gisela, Sabine Ruß und Christoph M. Haas: Zweite Kammern, 2. überarbeitete und erweiterte Auflage, Oldenbourg Wissenschaftsverlag GmbH, München 2010, 576 Seiten, $€ 49,80$.

Das Schattendasein, das viele Zweite Kammern sowohl in der öffentlichen als auch in der politikwissenschaftlichen Wahrnehmung führen, entspricht nicht zwangsläufig ihrer tatsächlichen Bedeutung im jeweiligen politischen System. Diese Einsicht gewinnt man aus beiden Handbüchern. Dennoch unterscheiden sich beide Bände deutlich - sowohl in ihrem Anspruch als auch in der Qualität von Darstellung und Analyse.

Der von Sven Leunig herausgegebene Band soll eine Forschungslücke schließen. Es handelt sich um die erste Darstellung, in der alle föderalen Zweiten Kammern vergleichend betrachtet werden. Von diesem sehr breiten Ansatz - immerhin werden die 20 Zweiten Kammern aller Bundesstaaten weltweit untersucht, einschließlich eines Exkurses auf den im Jahr 2000 abgeschafften Senat Venezuelas - verspricht sich der Herausgeber auch eine 
empirisch ausreichend gesättigte Grundlage, um zuverlässige Aussagen über Zusammenhänge zwischen dem Typ von Regierungssystem und den Ausprägungen des föderalen Systems zu formulieren. Dadurch, dass auch die Zweiten Kammern solcher Staaten dargestellt werden, die - wenn überhaupt - höchstens als "defekte“ Demokratien eingestuft werden könnten (zum Beispiel Nigeria, Pakistan, Malaysia), sind - so zumindest Leunig - auch Rückschlüsse darauf möglich, ob föderale Elemente geeignet sind, multiethnische Staaten zu stabilisieren. Dieser anspruchsvollen Zielsetzung wird das Buch, an dem überwiegend Nachwuchswissenschaftler (einzelne Autoren waren zum Zeitpunkt der Abfassung der Beiträge sogar noch im Studium) beteiligt waren, jedoch nur bedingt gerecht. Zwar sind neben dem lesenswerten Fazit Leunigs auch in anderen Kapiteln aufschlussreiche Zusammenhänge zu finden; das Erscheinungsbild des Bandes leidet aber zunächst unter der ungenügenden Redaktion: Immer wieder stören orthografische und grammatikalische Fehler (zum Beispiel Philipp Höfer über Kanada), wird ein wörtliches Zitat zweifach angeführt (Daniel Gerstenhauer über Bosnien-Herzegowina), bleiben redaktionelle Hinweise auch in der Druckfassung stehen (Stefanie Seifert über Pakistan). Hinzu treten inhaltliche Schwächen: Zuweilen fehlt die fachliche Substanz - sei es, weil die Daten- und Materialbasis offenbar zu dünn war (Pakistan), sei es, weil die politikwissenschaftlich interessanten Fragen gegenüber verfahrenstechnischen Details deutlich zu kurz kommen. So verweist Eva Range in ihrem Beitrag über den südafrikanischen Nationalrat der Provinzen (National Council of Provinces, NCOP) auf die Literatur, nach deren Einschätzung der NCOP in Organisation wie Funktion deutliche Ähnlichkeit zum deutschen Bundesrat aufweise. Dieser Aspekt, der sich mit der für einen komparativ angelegten Band naheliegenden Frage hätte verbinden lassen, ob diese Einschätzung erstens tatsächlich zutrifft und ob sie gegebenenfalls zweitens zur Folge hat, dass das Bundesratsmodell im südafrikanischen Föderalismus ähnliche Auswirkungen hat wie im bundesdeutschen, wird im Weiteren jedoch nur noch kursorisch (bei den Provinzdelegationen für den NCOP, die sich aus Mitgliedern der Exekutive und der Legislative zusammensetzen) thematisiert. Die Entscheidung des Herausgebers, auch den pakistanischen Senat zu behandeln, überzeugt nicht: Schließlich kann der von Stefanie Seifert gewählte Rückgriff auf die ausgiebige Nennung einschlägiger Verfassungsartikel und Geschäftsordnungsregeln schon deshalb keine adäquate Reaktion auf die unzureichende Literaturlage sein, weil „eine Arbeit des Senats, wie sie im Folgenden dargestellt wird, in den vergangenen 35 Jahren häufig nicht möglich war" (S. 187). Mit der politischen Wirklichkeit in Pakistan hat die dennoch recht ausführliche Darstellung des dortigen Senats demnach nur wenig zu tun.

Leunigs Fazit leistet hingegen deutlich mehr als eine schlussfolgernde Zusammenfassung. Hier wird tatsächlich strukturiert und kategorisiert sowie bedenkenswerte Einschätzungen zur Stärke beziehungsweise Schwäche Zweiter Kammern mit Blick sowohl auf ihre Funktionen als auch auf ihre parteipolitische Durchdringung geliefert. Leunig nutzt seine Schlussbetrachtungen auch für eine inhaltliche Auseinandersetzung mit Roland Sturms (2002) (zum Teil gemeinsam mit Suzanne S. Schüttemeyer, 1992) Überlegungen zu föderalen Zweiten Kammern. Während Sturm und Schüttemeyer laut Leunig vor allem die Funktion der Vertretung regionaler Interessen und deren Nichtwahrnehmung als Indiz für die Schwäche einer Zweiten Kammer anführten, stellt er vor dem Hintergrund der Ergebnisse im vorliegenden Band die Eignung dieses Kriteriums in Zweifel. Es „muss bestritten werden, dass es tatsächlich die primäre Aufgabe Zweiter Kammern in föderalen Staaten ist, regionale Interessen zu vertreten“ (S. 299). Hier handele es sich vielmehr um eine „modernere Erschei- 
nung“, die sich erst im Grundgesetz und nachfolgenden Verfassungsregelungen wie etwa in der Russländischen Föderation (1993) und Südafrika (1996) finde. Abgesehen von dieser etwas bemüht wirkenden Abgrenzung kommt aber auch Leunig - unter nachfolgendem aufschlussreichen Bezug auf die unterschiedlichen Länderbeispiele - zu dem Ergebnis, dass die politische Zukunft einer Zweiten Kammer immer dann prekär ist, wenn sie überhaupt keine erkennbare Funktion im jeweiligen System wahrnehmen kann, also weder Artikulationsorgan föderaler Interessen ist, noch gewaltenhemmend wirkt oder die Rolle eines „sanior pars" oder einer „chambre de réflexion“ einnimmt.

In dem von Gisela Riescher, Sabine Ruß und Christoph M. Haas herausgegebenen Sammelband liegt der Fokus nicht allein auf föderalen Zweiten Kammern, von denen zehn untersucht werden, sondern auch solche in unitarischen Systemen (acht Beispiele) sowie drei abgeschaffte Zweite Kammern werden portraitiert. Diese Entscheidung zur Weitung der Perspektive lässt sich systematisch begründen; schließlich fördert sie die Erkenntnis, dass Zweite Kammern „auch ohne die in der Bikameralismusforschung so stark gewichtete territoriale Repräsentationsbasis ein hohes Maß an funktionaler Relevanz erreichen " (S. 517). Auch wenn zum Beispiel der Französische Senat keine föderal begründete Kammer sei, fungiere auch er als territoriales Repräsentationsorgan und könne als Vertretungsorgan der „ländlichen Räume“ gelten.

Auch in diesem Band sind alle Einzelbeiträge nach einem einheitlichen Muster aufgebaut. Obwohl in zweiter Auflage erschienen (Erstauflage 2000), handelt es sich nicht um eine bloße Fortschreibung und Aktualisierung, sondern überwiegend um eine vollständige Überarbeitung beziehungsweise sogar Neufassung der Beiträge. Dieses Vorgehen ist auch dem Umstand geschuldet, dass nahezu alle bikameralen Systeme in den letzten Jahren deutliche Veränderungen erfahren haben, sei es durch Verfassungsreformen, sei es durch Strukturänderungen im Parteiensystem. Diesen Reformen, ihren Motiven und Perspektiven räumen die einzelnen Beiträge relativ viel Platz ein. Ausgesprochen sinnvoll - nicht nur für Studierende, die von der klaren Strukturierung dieses Lehrbuchs begeistert sein dürften erscheint das Einführungskapitel von Christoph M. Haas, in dem er nicht nur Begriffe und die Entstehungsgeschichte Zweiter Kammern erklärt und gleich ihr Hauptproblem - die Existenzproblematik - anspricht, sondern mit Hilfe recht anschaulicher Typologien einen ersten Zugang zum Thema ermöglicht. Sowohl die Gesamtanlage dieses Bands als auch die einzelnen Beiträge erscheinen insgesamt systematischer und klarer strukturiert als das „Handbuch Föderale Zweite Kammern“, bei dem zu häufig der Schilderung von Detailregelungen der Vorzug vor den großen Linien und der politikwissenschaftlich relevanten Analyse gegeben wurde. Leserfreundlich sind darüber hinaus die im Anhang enthaltenen Übersichten über die Staaten der Welt und ihre gegebenenfalls vorhandene Zweite Kammer, die Kurzübersicht über deren Struktur sowie das Glossar, das auch die Funktion eines (das ebenfalls vorhandene Personenregister ergänzenden) Sachregisters erfüllt.

Auch der Band „Zweite Kammern“ schließt an die Behandlung von insgesamt 21 Fallbeispielen ein abschließendes Analysekapitel (Riescher / Ruß) an. Dieses greift aber weniger die vor allem auf institutionelle Aspekte bezogenen Gliederungsschemata der Einzelbeiträge in komparativer Perspektive auf, sondern bemüht sich um eine zusätzliche Dimension - die Einbindung einer politics-orientierten Betrachtung sowie einen Ausblick auf weitere Forschungsansätze. Die Frage nach der Bedeutung Zweiter Kammern für den jeweiligen politischen Entscheidungsprozess führt zu ihrer „dominanten Funktionslogik“, und zwar sowohl in föderalen als auch in unitarischen Systemen: dem Kompromiss. Dieser sei nicht 
nur die „strukturelle Ursprungsformel“ Zweiter Kammern, sondern auch die „funktionelle Schüsselformel“ für ihre Bedeutung im politischen Prozess. Für ihren Vergleich der Funktion unterschiedlich situierter Zweiter Kammern greifen Riescher und Ruß auf den Vetospieleransatz von George Tsebelis zurück und arbeiten die „funktionale Relevanz Zweiter Kammern zur Durchsetzung verhandlungsdemokratischer Mechanismen und zur Abschwächung des Mehrheitsprinzips“ heraus. Ihr Hinweis, dass es neben den normierten auch potentielle Funktionen sind, die einer Zweiten Kammer zur Akzentsetzung offen stehen (wie etwa die Herstellung von Gegenöffentlichkeit), erschließt eine grundsätzliche Aufgabe Zweiter Kammern, nämlich „Agenda-Setter zu kontrollieren“ (S. 514). Auf diese Weise könnten sie erstens bereits bei der Bestimmung von Themen ein Verhandlungselement einführen und es zweitens einem Agenda-Setter zumindest schwerer machen, mit seiner Formulierung eines Gesetzesvorhabens auch gleich die Antwort vorzustrukturieren. Doch selbst bei dieser bescheiden anmutenden Funktionszuweisung gibt es - so die Autorinnen - Zweite Kammern, die auch dann keine nennenswerten Legislativfunktionen aufzuweisen haben. Während früher auch das Japanische Oberhaus in diese Kategorie gehörte, konnte dieses in den letzten Jahren seine Position ausbauen. Damit findet sich inzwischen allein der Österreichische Bundesrat in der Kategorie einer Zweiten Kammer ohne erwähnenswerte Gesetzgebungsfunktion.

Es passt in das Gesamtbild, das die beiden Bände beim Leser hinterlassen, dass der analytische Gehalt des Schlusskapitels in dem von Riescher / Ruß / Haas herausgegebenen Handbuch deutlich weiter reicht als das Gegenstück von Sven Leunig. Zweifelsohne verspricht die Lektüre beider Bücher einen Gewinn; bei Ressourcenknappheit kann aber eine Präferenz ausgesprochen werden: „Zweite Kammern“ von Riescher / Ruß / Haas lohnt sich nicht nur für diejenigen, die ohnehin an bikameralen Systemen interessiert sind.

Ursula Münch

\section{Landesverfassungsgerichte: Einfluss politologisch untersucht}

Flick, Martina: Organstreitverfahren vor den Landesverfassungsgerichten. Eine politikwissenschaftliche Untersuchung, Peter Lang Verlag, Frankfurt am Main u.a. 2011, 303 Seiten, $€ 56,60$.

Martina Flick untersucht in ihrer Dissertation aus politikwissenschaftlicher Sicht den Einfluss der Landesverfassungsgerichte auf den politischen Prozess in den Ländern. Ihre Arbeit entstand im Rahmen des Forschungsprojekts „Demokratiemuster in den deutschen Bundesländern und ihre politischen, gesellschaftlichen und ökonomischen Auswirkungen “, das an der Universität Konstanz von den Professoren Adrian Vatter und Markus Freitag geleitet wurde; beide haben die Doktorarbeit auch betreut.

Es ist sehr zu begrüßen, dass sich die Politikwissenschaft der föderalen Vielfalt der politischen Systeme der Bundesländer widmet, um Gemeinsamkeiten und Unterschiede zu untersuchen sowie Akteure und ihre Beziehungen in den Blick zu nehmen. Im Hinblick auf den Einfluss der Landesverfassungsgerichte ist die Analyse der Organstreitverfahren ein viel versprechender Anknüpfungspunkt, denn es geht in Streitigkeiten zwischen obersten 